\title{
Cardiac rehabilitation in Austria: Iong term health-related quality of life outcomes
}

\author{
Stefan Höfer*1, Werner Kullich², Ursula Graninger ${ }^{3}$, Manfred Wonisch', \\ Alfred Gaßner ${ }^{5}$, Martin Klicpera ${ }^{6}$, Herbert Laimer ${ }^{7}$, Christiane Marko ${ }^{8}$, \\ Helmut Schwann ${ }^{9}$ and Rudolf Müller ${ }^{3}$
}

\begin{abstract}
Address: ${ }^{1}$ Medical University Innsbruck, Department of Medical Psychology, Innsbruck, Austria, ${ }^{2}$ Ludwig-Boltzmann-Cluster, Institute for Rehabilitation, Saalfelden, Austria, ${ }^{3}$ Austrian Pension Insurance Institution, Pensionsversicherungsanstalt, Vienna, Austria, ${ }^{4}$ Center for Cardiac Rehabilitation, St. Radegund, Austria, ${ }^{5}$ Center for Cardiac Rehabilitation, Großgmain, Austria, ${ }^{6}$ Center for Cardiac Rehabilitation, Hochegg, Austria, ${ }^{7}$ Center for Cardiac Rehabilitation, Bad Tatzmannsdorf, Austria, ${ }^{8}$ Center for Cardiac Rehabilitation, Felbring, Austria and ${ }^{9}$ Center for Cardiac Rehabilitation, Saalfelden, Austria

Email: Stefan Höfer* - stefan.hoefer@i-med.ac.at; Werner Kullich - lbirehab@aon.at; Ursula Graninger - ursula.graninger@pva.sozvers.at; Manfred Wonisch - manfred.wonisch@pva.sozvers.at; Alfred Gaßner - alfred.gassner@pva.sozvers.at;

Martin Klicpera - martin.klicpera@pva.sozvers.at; Herbert Laimer - herbert.laimer@pva.sozvers.at;

Christiane Marko - christiane.marko@pva.sozvers.at; Helmut Schwann - helmut.schwann@pva.sozvers.at;

Rudolf Müller - rudolf.mueller@pva.sozvers.at

* Corresponding author
\end{abstract}

Published: 8 December 2009

Health and Quality of Life Outcomes 2009, 7:99 doi:10.1 186/1477-7525-7-99

This article is available from: http://www.hqlo.com/content/7///99

(C) 2009 Höfer et al; licensee BioMed Central Ltd.

This is an Open Access article distributed under the terms of the Creative Commons Attribution License (http://creativecommons.org/licenses/by/2.0), which permits unrestricted use, distribution, and reproduction in any medium, provided the original work is properly cited.
Received: 13 May 2009

Accepted: 8 December 2009

\begin{abstract}
Background: The goal of cardiac rehabilitation programs is not only to prolong life but also to improve physical functioning, symptoms, well-being, and health-related quality of life (HRQL). The aim of this study was to document the long-term effect of a I-month inpatient cardiac rehabilitation intervention on HRQL in Austria.

Methods: Patients ( $N=487,64.7 \%$ male, age $60.9 \pm 12.5$ SD years) after myocardial infarction, with or without percutaneous interventions, coronary artery bypass grafting or valve surgery underwent inpatient cardiac rehabilitation and were included in this long-term observational study (two years follow-up). HRQL was measured with both the MacNew Heart Disease Quality of Life Instrument [MacNew] and EuroQoL-5D [EQ$5 \mathrm{D}]$.

Results: All MacNew scale scores improved significantly $(p<0.00 \mathrm{I})$ and exceeded the minimal important difference ( $0.5 \mathrm{MacNew}$ points) by the end of rehabilitation. Although all MacNew scale scores deteriorated significantly over the two year follow-up period $(p<.001)$, all MacNew scale scores still remained significantly higher than the pre-rehabilitation values. The mean improvement after two years in the MacNew social scale exceeded the minimal important difference while MacNew scale scores greater than the minimal important difference were reported by $40-49 \%$ of the patients.
\end{abstract}

Two years after rehabilitation the mean improvement in the EQ-5D Visual Analogue Scale score was not significant with no significant change in the proportion of patients reporting problems at this time.

Conclusion: These findings provide a first indication that two years following inpatient cardiac rehabilitation in Austria, the long-term improvements in HRQL are statistically significant and clinically relevant for almost $50 \%$ of the patients. Future controlled randomized trials comparing different cardiac rehabilitation programs are needed. 


\section{Background}

Besides prolonging life, the objectives of cardiac rehabilitation (CR) include the reduction of symptoms and the improvement of physical functioning and general wellbeing $[1,2]$. These outcomes are typically considered to be patient-reported outcomes (PRO) and have top-tier priority when it comes to assessing quality in cardiovascular care [3]. A recently published meta-analysis showed that 12 out of 12 exercise-based outpatient CR programs improved health-related quality of life (HRQL) but the magnitude of improvement in HRQL with cardiac rehabilitation exceeded that of the controls in only two trials [4].

There is a great variety of CR programs in the different European countries with either inpatient or outpatient (including home-based CR [5]) or both CR programs available for patients [6]. A wide range of patients having undergone different interventions (e.g. percutaneous coronary intervention (PCI) and coronary artery bypass grafting (CABG) or heart valve surgery (HVS)) and presenting various diagnoses (myocardial infarction (MI), angina or heart failure (HF)) are eligible for these programs which makes it difficult to compare PROs. Inpatient as well as outpatient CR programs are provided in Austria [7] and there is evidence from a non-randomized study that both types of CR programs adequately improve the short term (3-month) outcome of HRQL [8]. In addition, short-term studies including PRO and clinical data for the major six Austrian cardiac inpatient rehabilitation centers have documented statistically significant and clinical important improvements in HRQL and reduction of risk factors in an unselected patient group [9].

Although the Austrian legal framework makes it mandatory that the health care systems and their long-term benefits are evaluated from a patient-centered perspective (Gesundheitsqualitätsgesetz BGBL I Nr. 179/2004), there is little or no data available regarding the long-term $(>12$ months) effects of inpatient CR programs on HRQL [10]. Further, the question about which particular sub-group within the population of eligible patients enjoys the greatest benefits from these programs within a particular timeframe and in accordance with national $[11,12]$ or international guidelines [13] has not been answered. The aim of this study was therefore to document the long-term $\mathrm{PRO}$ improvements of the inpatient CR programs available in Austria.

\section{Methods}

Over a period of 8 weeks in 2004, 487 consecutive patients after MI, angina or heart valve disease with or without PCI, CABG or heart valve surgery in six cardiac rehabilitation centers managed by the Austrian Pension
Insurance Institution ("Pensionsversicherungsanstalt" or "PVA") were included in this observational study. Patients completed the 4-week inpatient CR program as soon as possible after initial treatment. A detailed description of the CR program has been published and the selected patient group constitutes a representative sample of the participants in the inpatient CR programs available in Austria [9]. The protocol was approved by the institutional review board of the Austrian Pension Insurance Institution.

Baseline data were collected at the beginning (pre rehabilitation, t0) and at the end of the 4 week inpatient CR (post rehabilitation, one month t1) [9]. The two year follow-up was performed as a postal follow-up ( $\mathrm{t} 2$ ). The mailed package included a prepaid return envelope with the two questionnaires used at baseline, the MacNew Heart Disease Health-related Quality of Life Instrument [MacNew] and the EuroQol 5D [EQ-5D] plus a list of major adverse cardiac events. One postal reminder was sent out if patients did not return the initial questionnaire.

\section{MacNew}

The MacNew is an internationally well documented valid and reliable instrument to assess HRQL for patients with different manifestations of heart disease, such as angina pectoris [14], myocardial infarction [15], heart failure [16], and arrhythmia [17] as well as different interventions (such as PCI, CABG [18], pacemaker implant [19] or CR $[5,8,20])$. Currently the MacNew is the only international disease-specific HRQL instrument that ensures a reliable and valid assessment and comparison of cardiovascular patients with varying presentations and symptoms of their disease.

The MacNew comprises 27 items which are scored from 1 (poor HRQL) to 7 (high HRQL) and consists of three scales: physical limitations, emotional function, and social function; additionally an overall HRQL score can be calculated [21]. Reference data are available for different diagnostic entities and age groups [22]. The minimal important difference (MID; knowledge of the smallest change in instrument score that patients perceive as important [23]) for a MacNew change score has been established to be 0.5 MacNew points [22].

\section{EQ-5D}

The EQ-5D is a generic instrument for the measurement of HRQL and therefore particularly suited for comparisons with other diseases (e.g. cancer). On the basis of the utility approach, the EQ-5D can be used to calculate quality adjusted life years (QALYs) [24]. The EQ-5D consists of a 5-dimensional descriptive system and a visual analogue scale allowing assessment of relevant segments of HRQL: 
mobility, self-care, usual activities, pain/discomfort, and anxiety/depression. The EQ-5D has repeatedly been used and was validated with the aid of the MacNew in Germanspeaking CHD patients with acceptable psychometric properties (test-retest reliability and responsiveness) [25].

\section{Major adverse cardiac events}

The patients were queried whether and when the following major adverse cardiac events had occurred in the last two years: 1) heart attack, 2) symptoms of angina, 3) bypass surgery, 4) valve replacement, and 5) coronary intervention. In addition, the patients were asked if they had participated in other rehabilitation interventions such as another inpatient rehabilitation program or a follow-up outpatient rehabilitation program. Death as a major adverse cardiac event was recorded with the help of the Austrian health information system.

\section{Statistical analysis}

Descriptive procedures (means, standard deviation, frequencies) were used to describe patient characteristics. To compare responders with non-responders independent ttest and chi-square were used. Paired t-test (MacNew) and Wilcoxon (EQ-5D) test were applied to check the statistical significance for time and analysis of variance for group comparisons.

Effect sizes for the comparison baseline/follow-up were calculated $(\mathrm{ES}=(\mathrm{M} 1-\mathrm{M} 2) / \mathrm{SD} 1)$. Values between 0.20 and $<0.50$ are considered as small, values between 0.50 and $<$ 0.80 as moderate and $\geq 0.80$ as high [26]. The significance level was established at $\mathrm{p}<0.05$. All analyses were conducted using the statistics software package SPSS 16 for Windows (SPSS Incorp., USA).

\section{Results}

Questionnaires were returned by 351 patients (mean age of $60.9 \pm 12.5$ years, $66 \%$ males, completion rate of $72.1 \%$, Table 1). Additional selected baseline sociodemographic and clinical variables for all responders and non-responders are given in Table 1. Reasons for not returning the questionnaires included death $(14.7 \%)$, incorrect address (5.2\%), and unknown (80.1\%). Compared to responders, non-responders were 2.5 times more likely to have been working at baseline (Table 1). Based on the other available variables no significant difference between responders and non-responders could be detected.

During the two year follow-up, major adverse cardiac events among the 487 patients were recorded on 140 occasions. It included 20 deaths (4.1\%) with angina the most frequent event (11.9\%, Table 2). A single major adverse cardiac event was recorded for 76 patients while 21 patients reported more than one.
There was a significant short-term improvement in all MacNew HRQL scales over the one-month inpatient CR program (Table 3, and [9]) with fewer patients ( $p<0.001)$ reporting problems on the EQ-5D mobility, daily activities and pain/discomfort sub-scales at the end of inpatient CR (Table 7 in [9]). It is important to note that both responders and non-responders reported the same initial improvement in all MacNew and EQ-5D HRQL scale scores after CR (global: $\mathrm{p}=.622$; physical: $\mathrm{p}=.948$; emotional: $\mathrm{p}=.377$; social: $\mathrm{p}=.711$, mobility; $\mathrm{p}=.784$; selfcare: $\mathrm{p}=.881$; daily activities: $\mathrm{p}=.451$; pain: $\mathrm{p}=.655$; anxiety/depression: $\mathrm{p}=.293$ ).

Over the two year period following the end of inpatient rehabilitation program, HRQL significantly decreased in all MacNew scales (e.g., global MacNew, Figure 1). However, at the two year follow-up the mean HRQL for all MacNew scale scores were still significantly higher than at baseline with the social HRQL on average still above the MID of 0.5 MacNew points (change in global HRQL: 0.33 $\mathrm{p}=<.001$; physical HRQL: $0.35 \mathrm{p}=<.001$; emotional HRQL: $0.24 \mathrm{p}=.003$; social HRQL: $0.52 \mathrm{p}=<.001)$. Although $43.0 \%$ [ $n=151]$ of the patients reported an improvement in HRQL over the two-year follow-up that was equal to or exceeded the MID of 0.5 points, the effect sizes were small two years after inpatient CR (Table 3).

On the basis of the MacNew MID, the responders were then grouped as having a negative (-0.5 MacNew points), unchanged [-0.49-0.49 MacNew points) or positive $[+0.5$ MacNew points] MID (Table 3). About 25\% of the patients reported either a clinically important deterioration or remained unchanged with between 40 and $49 \%$ of the patients reporting a clinically important improvement on the MacNew HRQL scale scores. Moreover, patients who reported an improved HRQL of greater than the MID two years after CR had initially reported an improvement of 1.12 MacNew points with CR. This initial improvement was significantly higher $(\mathrm{p}<0.001)$ than that reported by either those whose HRQL had deteriorated within two years (initial improvement $=0.38$ MacNew points) or those whose HRQL remained unchanged over two years (initial improvement $=0.42 \mathrm{MacNew}$ points).

On the basis of the change in MacNew global HRQL, the effect sizes and the proportion of patients exceeding the MacNew MID of 0.5 points were calculated for the responders in accordance with the main diagnosis, the pre-treatment, the risk profile, and the socio-demographic status (age groups and gender) (Additional file 1; Table S1). HRQL improved at two years in patients with ischaemic heart disease $(\mathrm{p}<0.002)$ with a medium effect size of 0.65 and an improvement greater than the MID in $43.5 \%$; HRQL also improved in patients with heart valve disease $(\mathrm{p}=0.011)$ with a small effect size of 0.42 and an 
Table I: Clinical- and socio-demographic patient characteristics of responders and non-responders $(N=487)$

\begin{tabular}{|c|c|c|c|c|c|c|c|c|}
\hline \multirow[b]{2}{*}{ Variable } & \multirow[b]{2}{*}{ Categories } & \multicolumn{3}{|c|}{ Responder } & \multicolumn{3}{|c|}{ Non-responder } & \multirow[b]{2}{*}{ p-value } \\
\hline & & $M \pm S D$ & $\mathbf{N}$ & $\%$ & $M \pm S D$ & $\mathbf{N}$ & $\%$ & \\
\hline Age & & $60.9 \pm 12.5$ & & & $59.2 \pm 13.5$ & & & $0.08 \$$ \\
\hline \multirow[t]{3}{*}{ Gender } & Male & & 232 & $66.1 \%$ & & 95 & $70.8 \%$ & \\
\hline & Female & & 119 & $33.9 \%$ & & $4 I$ & $29.2 \%$ & \\
\hline & Total & & 351 & $100 \%$ & & 136 & $100 \%$ & $0.33 \S$ \\
\hline \multirow[t]{6}{*}{ Professional status } & Employed & & 43 & $12.3 \%$ & & 42 & $30.9 \%$ & \\
\hline & Retired & & 260 & $74.1 \%$ & & 63 & $46.3 \%$ & \\
\hline & Unemployed & & 9 & $2.6 \%$ & & 4 & $2.9 \%$ & \\
\hline & Other & & 25 & $7.1 \%$ & & 10 & $7.4 \%$ & \\
\hline & Total & & 337 & $96.0 \%$ & & 119 & $87.5 \%$ & \\
\hline & Missing & & 14 & $4.0 \%$ & & 17 & $12.5 \%$ & $0.08 \S$ \\
\hline \multirow[t]{5}{*}{ Education } & Compulsory education (CE) & & 101 & $28.8 \%$ & & 34 & $25.0 \%$ & \\
\hline & $C E+$ vocational training & & 135 & $38.5 \%$ & & 54 & $39.7 \%$ & \\
\hline & University degree & & 89 & $25.4 \%$ & & 32 & $23.5 \%$ & \\
\hline & Total & & 325 & $92.6 \%$ & & 120 & $88.2 \%$ & \\
\hline & Missing & & 26 & $7.4 \%$ & & 16 & $11.8 \%$ & $0.45 \S$ \\
\hline \multirow[t]{5}{*}{ Smoking status } & Current & & 39 & $11.1 \%$ & & 11 & $8.1 \%$ & \\
\hline & Ex-smoker & & 165 & $47.0 \%$ & & 69 & $50.7 \%$ & \\
\hline & Never smoker & & 119 & $33.9 \%$ & & 34 & $25 \%$ & \\
\hline & Total & & 323 & $92.0 \%$ & & 114 & 83.8 & \\
\hline & Missing & & 28 & $8.0 \%$ & & 22 & $16.2 \%$ & $0.34 \S$ \\
\hline \multirow[t]{3}{*}{ Primary diagnosis } & Ischemic heart disease & & 271 & $77.2 \%$ & & 109 & $80.1 \%$ & \\
\hline & Heart valve disease & & 51 & $14.5 \%$ & & 13 & $9.6 \%$ & \\
\hline & Other & & 29 & $8.3 \%$ & & 14 & $32.6 \%$ & $0.30 \S$ \\
\hline \multirow[t]{4}{*}{ Primary intervention } & $\mathrm{PCl}$ & & 170 & $48.4 \%$ & & 63 & $46.3 \%$ & \\
\hline & CABG & & 102 & $29.1 \%$ & & 39 & $28.7 \%$ & \\
\hline & HVS & & 54 & $15.4 \%$ & & 18 & $13.2 \%$ & \\
\hline & OPT & & 25 & $7.1 \%$ & & 16 & $11.8 \%$ & $0.77 \S$ \\
\hline NYHA\# & 1 & & 328 & $93.4 \%$ & & 118 & $86.8 \%$ & \\
\hline \multirow[t]{3}{*}{ (at discharge of initial CR) } & II & & 19 & $5.4 \%$ & & 16 & $11.8 \%$ & \\
\hline & III & & 4 & $1.1 \%$ & & 2 & $1.5 \%$ & $0.05 \S$ \\
\hline & IV & & - & - & & & & \\
\hline
\end{tabular}

$\mathrm{PCl}=$ percutaneous coronary intervention

$\mathrm{CABG}=$ coronary artery bypass grafting

HVS = heart valve surgery

OPT = optimal pharmacological treatment

\# New York Heart Association Classification

\$independent t-test

§chi-square

improvement greater than the MID in $49 \%$. As far as the treatment before CR is concerned, HRQL improved over the two years in patients with CABG and HVS $(\mathrm{p}<0.001)$ with medium effect sizes of 0.60 and 0.64 , respectively, and an improvement greater than the MID in $58.4 \%$ of the patients after CABG and in $60.6 \%$ of patients having undergone HVS.

Patients without hypertension, without diabetes or without hypercholesterolemia improved their HRQL $(\mathrm{p}<$
0.001 ) at two years with a small (no diabetes, ES $=0.38$ ) or medium effect sizes (no hypertension, $\mathrm{ES}=0.64$; no hypercholesterol, $\mathrm{ES}=0.56$ ) and an improvement greater than the MID two years after CR in $48-56 \%$ of the patients. Patients with no major adverse cardiac event in the last two years reported on average a medium effect, with $51 \%$ having a long lasting effect. In contrast, patients with one or more major adverse cardiac events deteriorated on average by $0.3 \mathrm{MacNew}$ points. It is interesting to note that these patients had already initially (t0) low 
Table 2: Major Adverse Cardiac Events in the last two years post cardiac rehabilitation

\begin{tabular}{lcc}
\hline Major Adverse Cardiac Event & Frequency N & Percentage \% \\
\hline Death (all-cause mortality) & 20 & $4.1 \%$ \\
Myocardial infarction & 10 & $2.1 \%$ \\
Recurring angina & 68 & $11,9 \%$ \\
CABG & 8 & $1.6 \%$ \\
Heart valve surgery & 8 & $1.6 \%$ \\
Coronary intervention (incl. stinting) & 26 & $5.3 \%$ \\
\hline Total & 140 & $28.7 \%$ \\
\hline
\end{tabular}

Multiple major adverse cardiac events in 21 patients

HRQL scale scores $(\mathrm{p}<0.001)$. Two years after CR, HRQL improved $(\mathrm{p}<0.001)$ in male but not in female patients, although the effect size was rather small $(\mathrm{ES}=0.37)$ despite the fact that $48 \%$ of the males reported an improvement greater than the MID.

Compared with mid-age (41-65) and older (>65) patients the young $(<41)$ showed strong effects, that were long lasting in $62 \%$ of the cases; although statistically significant effects could be shown for the age groups 41 and older, in terms of effect statistics there were no (age group 41-65) or small effects (age group 65+), with long lasting effects for $43-40 \%$ of the patients.

Beyond the often long lasting improvements in diseasespecific HRQL, no long-term improvements were observed in "mobility", "self-care" and "pain" as measured by the generic EQ-5D. The ability to perform usual daily life activities remained $6 \%$ higher two years after CR. However a considerable proportion of patients $(7 \%$ increase) reported some problems with "anxiety/depression" two years after CR (Table 4). The overall subjective health status based on the EQ-5D VAS Scale returned to baseline level (65.0).

\section{Discussion}

In this study, although mean HRQL decreased over the two years following CR in the 351 patients referred to the six participating Austrian inpatient rehabilitation centers, all MacNew scale scores HRQL remained significantly higher than at baseline with the mean social HRQL change greater than the MID of $0.5 \mathrm{MacNew}$ points. An additional indication for the positive long-term results of inpatient CR can be seen in the fact that as many as $60.1 \%$ of the patients reported an improved global MacNew HRQL score with $43.0 \%$ achieving or exceeding the MID. Comparing our results to published norm data, baseline HRQL values were below, t1 HRQL values were higher and t2 HRQL values were comparable to published norm values [22].

More detailed analyses made it possible to identify subgroups of patients who benefit most from the programs offered by the PVA inpatient CR centers in Austria. The improvement in HRQL showed greater effect sizes for patients with ischemic heart disease than for patients with valvular disease. Patients having undergone surgery (either CABG or HVS) prior to rehabilitation benefited more than patients after PCI which may be a consequence

Table 3: Mean change [M; 95\% Confidence Interval (95\% CI)] scores in MacNew HRQL, effect size statistics [ES; t0-t2] and minimal important difference [MID; t0-t2] over time

\begin{tabular}{|c|c|c|c|c|c|c|c|c|c|c|c|c|c|c|}
\hline \multirow[b]{2}{*}{ MacNew } & \multirow[b]{2}{*}{$\mathbf{N}$} & \multicolumn{2}{|l|}{ to-tl } & \multirow[b]{2}{*}{ p-value $\$$} & \multicolumn{2}{|l|}{ tl-t2 } & \multirow[b]{2}{*}{ p-value $\$$} & \multirow{2}{*}{$\begin{array}{c}\text { t0-t2 } \\
\text { ES }\end{array}$} & \multicolumn{2}{|c|}{$\begin{array}{l}\text { Deterioration } \\
\text { - MID [-0.5] }\end{array}$} & \multicolumn{2}{|c|}{$\begin{array}{l}\text { Unchanged } \\
\text { no change } \\
=\text { MID }\end{array}$} & \multicolumn{2}{|c|}{$\begin{array}{l}\text { Improvement } \\
\text { + MID }[0.5+]\end{array}$} \\
\hline & & $\mathbf{M}$ & $95 \% \mathrm{Cl}$ & & $\mathbf{M}$ & $95 \% \mathrm{Cl}$ & & & $\mathbf{N}$ & $\%$ & $\mathbf{N}$ & $\%$ & $\mathbf{N}$ & $\%$ \\
\hline Global & 340 & 0.7 & $0.60-0.85$ & $<0.001$ & -0.4 & $-0.60--0.23$ & $<0.001$ & 0.28 & 86 & 24.5 & 99 & 28.2 & $|5|$ & 43.0 \\
\hline Emotional & 320 & 0.6 & $0.49-0.76$ & $<0.001$ & -0.4 & $-0.61--0.19$ & $<0.001$ & 0.20 & 80 & 22.8 & 94 & 26.8 & 139 & 39.6 \\
\hline Physical & 339 & 0.9 & $1.0-0.70$ & $<0.001$ & -0.5 & $-0.72--0.33$ & $<0.001$ & 0.26 & 102 & 29.1 & 83 & 23.7 & 150 & 42.7 \\
\hline Social & 338 & 0.8 & $0.67-0.98$ & $<0.001$ & -0.3 & $-0.55--0.13$ & $<0.001$ & 0.40 & 85 & 24.2 & 77 & 21.9 & 171 & 48.7 \\
\hline
\end{tabular}

t0: baseline, pre rehabilitation

tl: post rehabilitation, one month

t2: two years follow-up

ES: effect size statistics from to to t2

MID: minimal important difference from to to t2 [MID $=0.5 \mathrm{MacNew}$ points]

\$paired t-test 


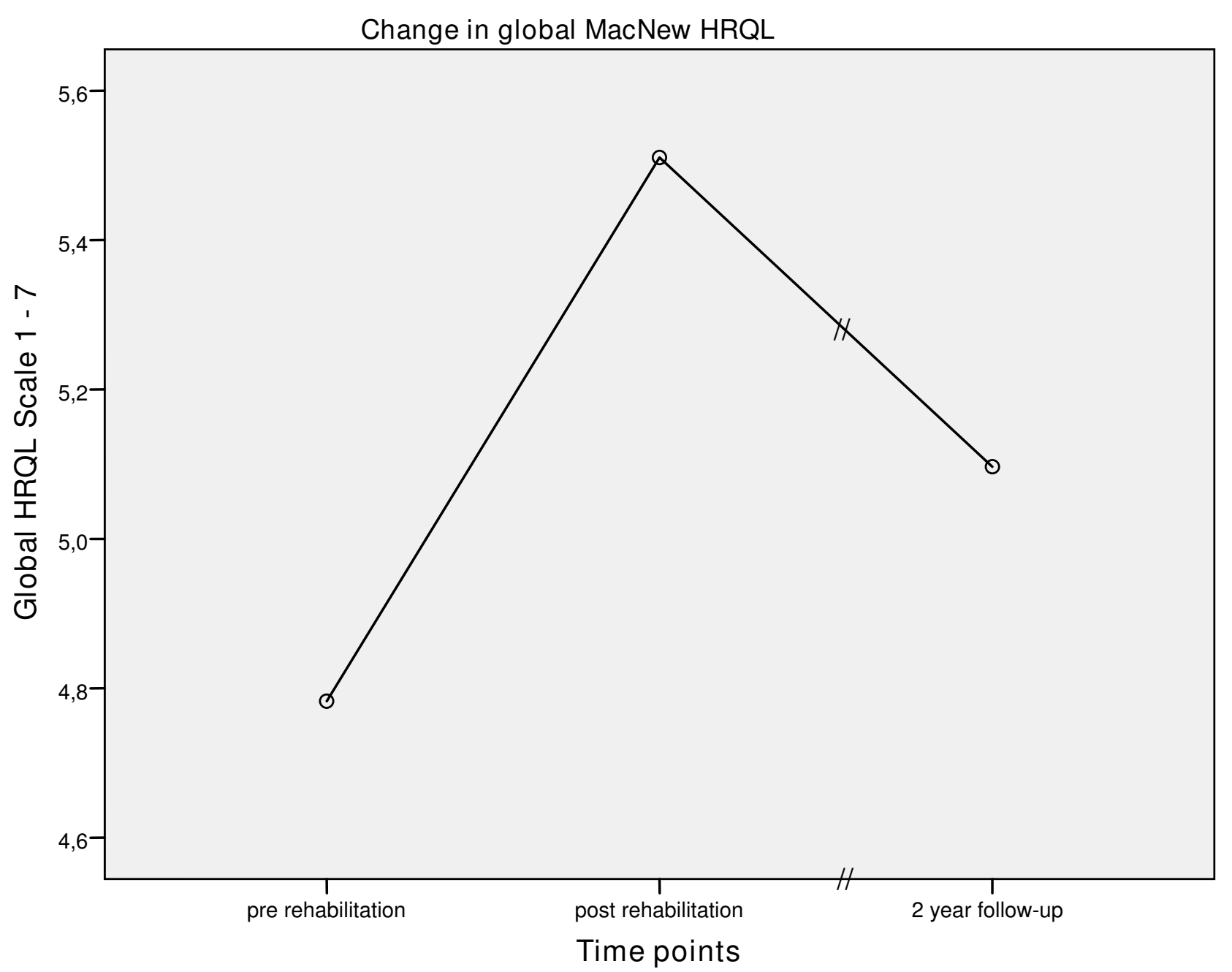

Figure I Change in mean global MacNew HRQL over time.

of the particular positive effect of the PCI that makes these patients report the greatest improvements in HRQL after a PCI but before rehabilitation [27]. This observation questions the additional benefit of inpatient CR as an opportunity for further improvement of HRQL in this patient group. This is in line with previous findings which have shown that CR is especially beneficial to CABG patients 12 months after CR (effect sizes i.e. 0.66 after 12 months) [10].

The management of patients with risk factors, i.e., smoking, hypertension, diabetes mellitus or hypercholesterolemia, where no long-term effects of statistical or clinical significance were observed, is a more challenging task. This raises the question whether it is possible to better manage patients at risk by providing additional on-going support (i.e. outpatient programs, long-term monitoring via modern media - eHealth) with the aim that this would bring about a potentially long-term benefit for these high risk patients.

The results of this study also suggest that younger patients, with an effect size of 0.91 , derive the greatest long-term benefit from inpatient CR in terms of an improved HRQL (with 63\% having a long-lasting effect). This is partly in line with previous findings, where increased age $(65+)$ was associated with mental HRQL comparable to community norms [28] or with a greater improvement after CR [10]. Our results, however, indicate that there is a U-form type of relationship. Either patients $<41$ or patients $65+$ years old reported small to medium effect sizes, with no effects for the age group 41-65. Finally, the fact that male 
Table 4: EQ-5D generic HRQL at baseline (to), one month ( $t \mathrm{l})$ and two years (t2) (percentage \%)

\begin{tabular}{|c|c|c|c|c|c|}
\hline \multicolumn{6}{|c|}{ Timepoint } \\
\hline & to & tl & $\mathbf{t 2}$ & $\mathbf{Z}$ & $p$-value $[\mathrm{t} 0-\mathrm{t} 2]$ \\
\hline \multicolumn{6}{|l|}{ EQ-5D Dimensions } \\
\hline \multicolumn{6}{|l|}{ Mobility $(N=291)$} \\
\hline No problems & $71.3 \%$ & $83.2 \%$ & $72 \%$ & & \\
\hline Some problems & $28.7 \%$ & $16.3 \%$ & $27.4 \%$ & & \\
\hline Severe problems & $0 \%$ & $0.5 \%$ & $0.6 \%$ & .630 & $.529 \$$ \\
\hline \multicolumn{6}{|l|}{ Self care $(N=293)$} \\
\hline No problems & $90.5 \%$ & $93.7 \%$ & $87.4 \%$ & & \\
\hline Some problems & $8.4 \%$ & $4.5 \%$ & $10.9 \%$ & & \\
\hline Severe problems & $1.1 \%$ & $1.8 \%$ & $1.8 \%$ & 1.376 & $.169 \$$ \\
\hline \multicolumn{6}{|c|}{ Daily activities $(N=290)$} \\
\hline No problems & $58.3 \%$ & $64.0 \%$ & $64.3 \%$ & & \\
\hline Some problems & $32.5 \%$ & $30.6 \%$ & $31.1 \%$ & & \\
\hline Severe problems & $9.2 \%$ & $5.4 \%$ & $4.6 \%$ & 1.999 & $.046 \$$ \\
\hline \multicolumn{6}{|l|}{ Pain $(N=291)$} \\
\hline No problems & $29.0 \%$ & $40.8 \%$ & $32.4 \%$ & & \\
\hline Some problems & $65.5 \%$ & $55.2 \%$ & $60.9 \%$ & & \\
\hline Severe problems & $5.5 \%$ & $4.1 \%$ & $.8 \%$ & .865 & $.387 \$$ \\
\hline \multicolumn{6}{|c|}{ Anxiety/depression $(\mathrm{N}=291)$} \\
\hline No problems & $65.9 \%$ & $68.9 \%$ & $60.9 \%$ & & \\
\hline Some problems & $31.0 \%$ & $28.6 \%$ & $35.8 \%$ & & \\
\hline Severe problems & $3.1 \%$ & $2.6 \%$ & $3.3 \%$ & 2.167 & $.030 \$$ \\
\hline \multicolumn{6}{|l|}{ VAS } \\
\hline Median $(\mathrm{N}=263)$ & 66.0 & 75.0 & 70.0 & & \\
\hline Mean $(N=208)$ & $63.7 \pm 17.6$ & $73.4 \pm 17.6$ & $65.0 \pm 20.4$ & & $.931 \#$ \\
\hline
\end{tabular}

VAS Visual Analogue Scale

\# T-test

\$Wilcoxon-Test

t0: baseline, pre rehabilitation

tl: post rehabilitation, one month

t2: two years follow-up

patients show a greater benefit than female ones may suggest a possible programmatic gender issue which needs further investigation.

The 5 dimensions of HRQL as measured by the generic EQ-5D did show improvements for daily activities for a small proportion of patients $(6 \%)$. However, in contrast to the disease-specific significant MacNew HRQL changes, the EQ-5D did not pick up an overall global health status improvement for the whole group, with values returning to pre-rehabilitation levels. This is in line with previous research demonstrating that disease specific instruments are more sensitive to change in contrast to generic HRQL instruments which are more useful when comparing different diagnoses [29]. Another important and more general finding is the reported increase in anxiety/depression two years after the end of the inpatient CR program. Since there is presently a controversial discussion about depression as a potential risk factor or a significant comorbidity [30-32] influencing the outcome [33], special attention needs to be paid to the diagnosis of anxiety/depression in patients attending inpatient CR [34].
Although patients with one or more major adverse cardiac events two years after CR reported the same initial improvement of $0.7 \mathrm{MacNew}$ points as patients without major adverse cardiac events in the follow-up period, their initial HRQL scale scores were significantly lower $(<4.5$ MacNew points) which means that the difference between the two groups was close to the MID of $0.5 \mathrm{MacNew}$ points. Low HRQL has been shown to have negative effects on adherence [35], and adherence itself is a highly relevant factor for health outcomes (e.g. $[36,37])$. In addition, a previous study using the MacNew scale scores predicted adverse cardiac events including death [38]. This corroborates the findings of the present study suggesting that initial screening for HRQL, especially at the beginning of $\mathrm{CR}$, may be a potential decision-making tool, to improve the identification of high risk patients. An intensive monitoring of high risk patients is advisable (i.e. with low initial HRQL: $<4.5 \mathrm{MacNew}$ points at the beginning of CR; or little HRQL improvement: $<0.5$ MID improvement) after the end of CR in order to prevent possible future major adverse cardiac events. Future studies and programs should address the benefit of ongoing brief con- 
tacts with patients having undergone CR with the aim of monitoring their health status with the help of modern eHealth technologies.

The amount of publications addressing PROs such as HRQL after inpatient cardiac rehabilitation is very limited. Published articles addressing inpatient cardiac rehabilitation primarily focus on cost analysis [39], cardiovascular risk factors $[40,41]$, consumer parameters (such as treatment satisfaction or patient expectations $[42,43])$ or its utilization $[44,45]$. In this study we documented the long-term (two years) HRQL outcome of patients following a one month inpatient CR. If HRQL has been used as an outcome parameter in cardiac rehabilitation evaluation studies a variety of measures have been used making outcome comparisons difficult [46]. For example Müller-Nordheim used in a similar study the generic SF-36 with a one year follow-up period. The general findings are comparable to ours, with large effect size HRQL improvements for patients after CABG, less after PCI and non after MI [10]. In contrast to our results, women reported more frequently improvements in health status then men. Overall, a consistent application of a single core heart disease specific HRQL outcome measure to allow program comparisons is warranted.

A major limitation of this study is the lack of a control group, which does not allow attributing the documented improvement in HRQL only to CR. As there is no evidence for the natural history of long-term recovery of patients not attending CR in Austria, it is difficult to distinguish the effect of CR from other factors. Further, although there is relatively little documentation of the long-term benefits of inpatient CR, it is a clinically well-established practice in Austria making the feasibility of randomization to a control versus an inpatient $\mathrm{CR}$ group questionable. In relation to this, a study by Benzer et al. comparing inpatient CR, outpatient CR and usual care (non-CR participants) over a short period of time indicated that there is a faster recovery of HRQL for CR attendees in contrast to no CR [8]. However the question of whether and how CR non-participants improve over a long term period such as two years remains unanswered and future evaluation projects need to consider the possibility of control groups at least for short-term outcomes (i.e. waiting-list controls). Further, the HRQL of non-responders (27.9\%) remains unclear. It should be noted, however, that the analyses of non-responders and responders did not suggest that there was a selection bias based on the available variables, or the initial HRQL improvements. Another limitation of this study is the lack of information about stroke as an additional major cardiac event.

This study documented improved HRQL for as many as $49 \%$ of all patients two years after CR, complementing the available literature on long term health outcomes after inpatient CR. In particular male patients up to 41 years with either ischemic heart disease or pre-treated with CABG or HVS and without risk factors benefited most from the existing CR programs. It may, therefore, be necessary to develop gender- and age-specific modules. HRQL screening for high risk patients (low HRQL) combined with a long term monitoring should be applied to minimize major cardiac events in high risk patients. Future controlled randomized trials comparing different cardiac rehabilitation programs using a single core heart disease specific PRO outcome measure are needed.

\section{Competing interests}

The authors declare that they have no competing interests.

\section{Authors' contributions}

SH drafted the manuscript and performed the statistical analysis. UG, WK and RM designed the study protocol. MW, AG, MK, HL, CM and HS organized and carried out the original study. All authors read and approved the final manuscript.

\section{Additional material}

\section{Additional file 1}

Table S1. Mean change [ $M \pm$ standard deviation, $S D]$, effect size statistics [ES; t0-t2] and minimal important difference [MID; t0-t2] over time in global MacNew HRQL scores according to subgroups.

Click here for file

[http://www.biomedcentral.com/content/supplementary/14777525-7-99-S1.DOC]

\section{Acknowledgements}

The authors are indebted to Professor N Oldridge for his helpful comments on this paper.

This project was internally funded by the Austrian Pension Insurance Institution.

\section{References}

I. Saner H: [From cardiac rehabilitation to prevention]. Wiener klinische Wochenschrift 2003, I I 5(2 I-22):743-744.

2. Committee WHO: Rehabilitation of patients with cardiovascular disease. Geneva: Technical report series 2701964.

3. Krumholz HM, Peterson ED, Ayanian JZ, Chin MH, DeBusk RF, Goldman L, Kiefe Cl, Powe NR, Rumsfeld JS, Spertus JA, Weintraub WS: Report of the National Heart, Lung, and Blood Institute working group on outcomes research in cardiovascular disease. Circulation 2005, I I I (23):3 I58-3 I66.

4. Taylor RS, Brown A, Ebrahim S, Jolliffe J, Noorani H, Rees K, Skidmore B, Stone JA, Thompson DR, Oldridge N: Exercise-based rehabilitation for patients with coronary heart disease: systematic review and meta-analysis of randomized controlled trials. The American journal of medicine 2004, I I 6( I 0):682-692

5. Dalal HM, Evans PH, Campbell JL, Taylor RS, Watt A, Read KL, Mourant AJ, Wingham J, Thompson DR, Pereira Gray DJ: Home-based versus hospital-based rehabilitation after myocardial infarction: A randomized trial with preference arms--Cornwall 
Heart Attack Rehabilitation Management Study (CHARMS). International journal of cardiology 2007, I I 9(2):202-2II.

6. Vanhees L, McGee HM, Dugmore LD, Schepers D, van Daele P: A representative study of cardiac rehabilitation activities in European Union Member States: the Carinex survey. J Cardiopulm Rehabil 2002, 22(4):264-272.

7. Benzer W, Mayr K, Abbuhl B: [Cardiac rehabilitation in Austria. A need to treat analysis]. Wiener klinische Wochenschrift 2003, I I 5(2 I-22):780-787.

8. Benzer W, Platter M, Oldridge NB, Schwann H, Machreich K, Kullich W, Mayr K, Philippi A, Gassner A, Dorler J, Hofer S: Short-term patient-reported outcomes after different exercise-based cardiac rehabilitation programmes. Eur J Cardiovasc Prev Rehabil 2007, I 4(3):44I-447.

9. Höfer S, Kullich W, Graninger U, Brandt D, Gassner A, Klicpera M, Laimer H, Marko C, Schwann H, Muller R: Cardiac rehabilitation in Austria: short term quality of life improvements in patients with heart disease. Wiener klinische Wochenschrift 2006 I I 8(23-24):744-753.

10. Muller-Nordhorn J, Kulig M, Binting S, Voller H, Gohlke H, Linde K, Willich $\mathrm{SN}$ : Change in quality of life in the year following cardiac rehabilitation. Qual Life Res 2004, 13(2):399-4I0.

II. Arbeitsgruppe Kardiologische Rehabilitation und Sekundärprävention der Österreichischen Kardiologischen Gesellschaft: Guidelines für die ambulante kardiologische Rehabilitation und Prävention in Österreich. Beschluß der Österreichischen Kardiologischen Gesellschaft vom April 2005. Journal für Kardiologie 2005, I 2( I I-I 2):303-309.

12. Brandt D: Editorial: Indikation zur Kardiologischen Rehabilitation. Journal für Kardiologie 2004, I I (II):435-436.

13. Graham I, Atar D, Borch-Johnsen K, Boysen G, Burell G, Cifkova R, Dallongeville J, De Backer G, Ebrahim S, Gjelsvik B, Herrmann-Lingen C, Hoes A, Humphries S, Knapton M, Perk J, Priori SG, Pyorala K Reiner Z, Ruilope L, Sans-Menendez S, Op Reimer WS, Weissberg P, Wood D, Yarnell J, Zamorano JL, Walma E, Fitzgerald T, Cooney MT, Dudina A, Vahanian A, et al.: European guidelines on cardiovascular disease prevention in clinical practice: executive summary. Fourth Joint Task Force of the European Society of Cardiology and other societies on cardiovascular disease prevention in clinical practice (constituted by representatives of nine societies and by invited experts). Eur J Cardiovasc Prev Rehabil 2007, I4(Suppl 2):EI-40.

14. Höfer S, Benzer W, Schüßler G, von Steinbüchel N, Oldridge NB: Health-related quality of life in patients with coronary artery disease treated for angina: validity and reliability of German translations of two specific questionnaires. Qual Life Res 2003 , I2(2): 199-2/2.

15. Höfer S, Benzer W, Brandt D, Laimer H, Schmidt P, Bernardo A, Oldridge NB: MacNew Heart Disease Lebensqualitätsfragebogen nach Herzinfarkt: die deutsche Version. Zeitschrift für Klinische Psychologie und Psychotherapie 2004, 33(4):270-280.

16. Höfer S, Schmid JP, Frick M, Benzer W, Laimer H, Oldridge N, Saner $\mathrm{H}$ : Psychometric properties of the MacNew heart disease health-related quality of life instrument in patients with heart failure. J Eval Clin Pract 2008, I 4(4):500-506.

17. Höfer S, Anelli-Monti M, Berger T, Hintringer F, Oldridge N, Benzer W: Psychometric properties of an established heart disease specific health-related quality of life questionnaire for pacemaker patients. Qual Life Res 2005, I4(8): 1937-1942.

18. Benzer W, Höfer S, Oldridge NB: Change in health-related quality of life following medical or invasive treatment for angina in patients with coronary artery disease. Herz 2003, 28(5):42। -428 .

19. Benzer W, Oldridge N, Anelli Monti M, Berger T, Hintringer F, Höfer S: Clinical predictors of health-related quality of life after pacemaker implantation. Wiener klinische Wochenschrift 2006, II 8(23-24):739-743.

20. Wisloff U, Stoylen A, Loennechen JP, Bruvold M, Rognmo O, Haram PM, Tjonna AE, Helgerud J, Slordahl SA, Lee SJ, Videm V, Bye A, Smith GL, Najjar SM, Ellingsen O, Skjaerpe T: Superior cardiovascular effect of aerobic interval training versus moderate continuous training in heart failure patients: a randomized study. Circulation 2007, II 5(24):3086-3094.

21. Höfer S, Lim LL, Guyatt GH, Oldridge NB: The MacNew Heart Disease health-related quality of life instrument: A summary. Health and quality of life outcomes 2004, 2(I):3.
22. Dixon T, Lim LL, Oldridge NB: The MacNew heart disease health-related quality of life instrument: reference data for users. Qual Life Res 2002, I I(2): 173-183.

23. Guyatt G, Schunemann H: How can quality of life researchers make their work more useful to health workers and their patients? Qual Life Res 2007, 16(7): $1097-1105$

24. Nord E: Methods for quality adjustment of life years. Social science \& medicine (1982) 1992, 34(5):559-569.

25. Schweikert B, Hahmann H, Leidl R: Validation of the EuroQol questionnaire in cardiac rehabilitation. Heart (British Cardiac Society) 2006, 92(I):62-67

26. Cohen J: A coefficient of agreement for nominal scales. Educational and Psychological Measurement 1960, XX(I):37-46.

27. Höfer S, Doering S, Rumpold G, Oldridge N, Benzer W: Determinants of health-related quality of life in patients with coronary artery disease. Eur J Cardiovasc Prev Rehabil 2006, I3(3):398-406.

28. Brown N, Melville M, Gray D, Young T, Munro J, Skene AM, Hampton JR: Quality of life four years after acute myocardial infarction: short form 36 scores compared with a normal population. Heart (British Cardiac Society) 1999, 8 I (4):352-358.

29. Testa MA, Simonson DC: Assesment of quality-of-life outcomes. The New England journal of medicine 1996, 334(I3):835-840.

30. Rozanski A, Blumenthal JA, Kaplan J: Impact of psychological factors on the pathogenesis of cardiovascular disease and implications for therapy. Circulation 1999, 99(16):2192-2217.

31. Yusuf S, Hawken S, Ounpuu S, Dans T, Avezum A, Lanas F, McQueen M, Budaj A, Pais P, Varigos J, Lisheng L: Effect of potentially modifiable risk factors associated with myocardial infarction in $\mathbf{5 2}$ countries (the INTERHEART study): case-control study. Lancet 2004, 364(9438):937-952.

32. Rugulies R: Depression as a predictor for coronary heart disease. a review and meta-analysis. Am J Prev Med 2002, 23(I):5I-6I.

33. Mayou RA, Gill D, Thompson DR, Day A, Hicks N, Volmink J, Neil A: Depression and anxiety as predictors of outcome after myocardial infarction. Psychosomatic medicine 2000, 62(2):2/2-219.

34. Rozanski A, Blumenthal JA, Davidson KW, Saab PG, Kubzansky L: The epidemiology, pathophysiology, and management of psychosocial risk factors in cardiac practice: the emerging field of behavioral cardiology. Journal of the American College of Cardiology 2005, 45(5):637-65I

35. Fogel J, Fauerbach JA, Ziegelstein RC, Bush DE: Quality of life in physical health domains predicts adherence among myocardial infarction patients even after adjusting for depressive symptoms. Journal of psychosomatic research 2004, 56(I):75-82.

36. Horwitz RI, Horwitz SM: Adherence to treatment and health outcomes. Archives of internal medicine 1993, I53(16): I863-I868.

37. Horwitz RI, Viscoli CM, Berkman L, Donaldson RM, Horwitz SM, Murray CJ, Ransohoff DF, Sindelar J: Treatment adherence and risk of death after a myocardial infarction. Lancet 1990 , 336(87 I 4):542-545.

38. Dixon T, Lim LL, Heller RF: Quality of life: an index for identifying high-risk cardiac patients. J Clin Epidemiol 200I, 54(9):952-960.

39. Zeidler ], Mittendorf T, Vahldiek G, Schulenburg JM von der: [Comparative cost analysis of outpatient and inpatient rehabilitation for cardiac diseases]. Herz 2008, 33(6):440-447.

40. Baberg HT, Uzun D, de Zeeuw J, Sinclair R, Bojara W, Mugge A, Schubmann R: [Health promotion and inpatient rehabilitation. Long-term effects of education in patients with coronary heart disease]. Herz 2005, 30(8):754-760.

4I. Voller H, Hahmann H, Gohlke H, Klein G, Rombeck B, Binting S, Willich SN: [Effects of inpatient rehabilitation on cardiovascular risk factors in patients with coronary heart disease. PINStudy Group]. Deutsche medizinische Wochenschrift (1946) 1999, 1 24(27):817-823.

42. Schubmann RM, Vogel H, Placzek T, Faller H: [Cardiac rehabilitation--expectations and appraisals of patients]. Die Rehabilitation 2005, 44(3): I 34- I 43.

43. Muthny FA, Berndt $H$, Gradaus D, Schweidtmann W: [Treatment satisfaction in cardiologic rehabilitation and attitude to various forms of rehabilitation]. Die Rehabilitation 1999, 38(2):72-78.

44. Spencer FA, Salami B, Yarzebski J, Lessard D, Gore JM, Goldberg RJ: Temporal trends and associated factors of inpatient cardiac rehabilitation in patients with acute myocardial infarction: a 
community-wide perspective. J Cardiopulm Rehabil 200I, $21(6): 377-384$.

45. Baessler A, Fischer M, Hengstenberg $C$, Holmer $S$, Hubauer $U$, Huf $V$, Mell S, Klein G, Riegger G, Schunkert H: [Inpatient rehabilitation improves implementation of therapeutic guidelines for secondary prevention in patients with coronary heart disease]. Zeitschrift fur Kardiologie 200I, 90(9):646-654.

46. McGee HM, Hevey D, Horgan JH: Psychosocial outcome assessments for use in cardiac rehabilitation service evaluation: a 10-year systematic review. Social science \& medicine (1982) 1999, 48(10): $1373-1393$.

Publish with Bio Med Central and every scientist can read your work free of charge

"BioMed Central will be the most significant development for disseminating the results of biomedical research in our lifetime. "

Sir Paul Nurse, Cancer Research UK

Your research papers will be:

- available free of charge to the entire biomedical community

- peer reviewed and published immediately upon acceptance

- cited in PubMed and archived on PubMed Central

- yours - you keep the copyright

Submit your manuscript here:

http://www.biomedcentral.com/info/publishing_adv.asp
BiolMedcentral 